\title{
Intra-Arterial Therapies for Metastatic Colorectal Cancer
}

\author{
David S. Wang, $\mathrm{MD}^{1} \quad$ John D. Louie, $\mathrm{MD}^{1} \quad$ Daniel Y. Sze, MD, $\mathrm{PhD}^{1}$ \\ ${ }^{1}$ Division of Interventional Radiology, Department of Radiology, \\ Stanford University Medical Center, Stanford, California \\ Address for correspondence David S. Wang, MD, Division of \\ Interventional Radiology, Department of Radiology, Stanford \\ Semin Intervent Radiol 2013;30:12-20 \\ University Medical Center, 300 Pasteur Drive, Room H3630, Stanford, \\ CA 94305-5642 (e-mail: dwang@stanfordmed.org).
}
Abstract
Keywords
- colorectal cancer
- liver metastases
- radioembolization
- drug-eluting beads
- irinotecan

Intra-arterial therapies for unresectable hepatic metastases from colorectal cancer include radioembolization (RE) with yttrium-90 microspheres, transarterial chemoembolization (TACE), hepatic arterial infusion, and percutaneous hepatic perfusion using an organ isolation system. In this article, we discuss our approach toward treatment selection, followed by details of how RE and TACE are performed at our institution.
Objectives: Upon completion of this article, the reader will be able to identify the role of intra-arterial therapies used in the treatment of colon cancer metastatic to the liver, as well as the indications and contraindications for the use of such therapies.

Accreditation: This activity has been planned and implemented in accordance with the Essential Areas and policies of the Accreditation Council for Continuing Medical Education through the joint sponsorship of Tufts University School of Medicine (TUSM) and Thieme Medical Publishers, New York. TUSM is accredited by the ACCME to provide continuing medical education for physicians.

Credit: Tufts University School of Medicine designates this journal-based CME activity for a maximum of 1 AMA PRA Category 1 Credit $^{\mathrm{TM}}$. Physicians should claim only the credit commensurate with the extent of their participation in the activity.

Colorectal cancer (CRC) is the second most commonly diagnosed cancer worldwide. ${ }^{1}$ In the United States, 20\% of these patients initially present with distant metastases, resulting in 5 -year survival rates in this subgroup $<12 \%{ }^{2}$ The liver is the most common and frequently dominant site of metastatic disease, occurring in 20 to $30 \%$ of patients at diagnosis with a prevalence up to $60 \%$ during the course of disease. ${ }^{3}$ Although surgical resection of the primary tumor and liver metastases is the most favorable treatment option (achieving 5-year survival rates of 37 to 58\%), only 10 to $25 \%$ of patients with hepatic metastases from CRC (hmCRC) are candidates for hepatic resection at initial presentation. ${ }^{4,5}$ In addition, of these, most patients develop recurrent disease after hepatic metastatectomy. For unresectable hmCRC patients, systemic chemotherapy remains the standard first-line therapy. Despite significant gains in therapeutic efficacy achieved in the past decade with modern systemic regimens that combine fluoropyrimidines and leucovorin with oxaliplatin (FOLFOX), irinotecan (FOLFIRI), and/or molecularly targeted biological agents such as bevacizumab (Avastin; Genentech, Inc., South San Francisco, CA), cetuximab (Erbitux; Bristol-Meyers Squibb Co., Princeton, NJ), panitumumab (Vectibix; Amgen, Inc., Thousand Oaks, CA), and regorafenib (Stivarga; Bayer HealthCare Pharmaceuticals, Wayne, NJ), ${ }^{6,7}$ most patients eventually develop progressive disease. There therefore remains a need for liver-directed locoregional therapies for patients with unresectable liverdominant or liver-only hmCRC, especially those who fail frontline systemic treatment regimens.

Hepatic intra-arterial therapies for hmCRC are intuitively attractive because the blood supply to liver metastases is almost exclusively via the hepatic arteries, whereas vascularization of normal liver parenchyma is $70 \%$ portal and 30\% arterial. ${ }^{8}$ Catheter-based hepatic intra-arterial therapies thus preferentially deliver therapeutic agents to metastatic foci while minimizing systemic exposure and treatment-associated toxicities. Contemporary intra-arterial treatment options for hmCRC include radioembolization (RE) with microspheres labeled with the $\beta$ emitter yttrium-90 ( ${ }^{90} \mathrm{Y}$ ); transarterial chemoembolization (TACE) using either emulsions of ethiodized oil and chemotherapy solution
Copyright (c) 2013 by Thieme Medical Publishers, Inc., 333 Seventh Avenue, New York, NY 10001, USA. Tel: +1(212) 584-4662.
DOI http://dx.doi.org/ 10.1055/s-0033-1333649. ISSN 0739-9529. 
(conventional TACE) or drug-eluting beads loaded with irinotecan (DEBIRI-TACE); hepatic arterial infusion (HAI) of chemotherapy; and percutaneous hepatic perfusion (PHP, also termed chemosaturation) using an organ isolation perfusion system with melphalan. ${ }^{9}$

\section{Treatment Triage and Rationale}

Relative to other hepatic intra-arterial therapeutic options, there is a more robust and mature body of evidence supporting the use of ${ }^{90} \mathrm{Y}$ RE to treat unresectable hmCRC patients. The data have consistently demonstrated clinical benefits and a low toxicity profile with this treatment regimen, with fatigue the most common adverse effect. Moreover, among metastatic processes, unresectable hmCRC has been the most extensively studied indication for RE. This collective clinical experience, resulting from phase 1 through 3 clinical trials that include prospectively randomized studies, is comprehensively summarized in recent reviews. ${ }^{10-14}$

Although several studies have shown early evidence that RE combined with systemic chemotherapy regimens potentiates treatment response compared with chemotherapy alone, ${ }^{15-20}$ further investigation is required to better define the role of RE as first- or second-line therapy in the treatment paradigm for hmCRC ( - Fig. 1). ${ }^{13}$ The currently available data, however, have established RE as a valuable therapeutic salvage option for patients who have failed prior systemic chemotherapy regimens (results of select studies are summarized in -Table 1). ${ }^{21-29}$ For example, in a multicenter prospective phase 2 trial of $50 \mathrm{hmCRC}$ patients who had failed prior oxaliplatin- and irinotecan-based chemotherapy regimens, the overall response rate after a single intra-arterial administration of ${ }^{90} \mathrm{Y}$ resin microspheres was $24 \%$, with disease stabilization in another $24 \%{ }^{21}$ Median overall survival was 16 months among responders, 8 months among nonresponders, and 13 months overall. Similarly, in a multicenter retrospective study of 208 unresectable hmCRC patients who had exhausted all other treatment options including systemic chemotherapy, ablation, and other locoregional intra-arterial therapies, overall response rate after RE with ${ }^{90} \mathrm{Y}$ resin microspheres was $35.5 \%$, with stable disease in $55 \%{ }^{22}$ Median overall survival in this study was 10.5 months among responders and 4.5 months in nonresponders. These results compare favorably with systemic salvage studies, where median overall survival rates range from 3 to 7 months. ${ }^{10,27}$ Because most unresectable hmCRC patients referred to our practice for hepatic intra-arterial therapy present in the salvage therapy setting, ${ }^{90} \mathrm{Y}$ RE has become our mainstay offering.

For unresectable hmCRC patients who have failed or have contraindications to RE, we selectively treat with DEBIRITACE. As a newer intra-arterial therapeutic option (with the first clinical experience reported in $2006^{30}$ ), the cumulative clinical data for DEBIRI-TACE remains limited, although the published results thus far are encouraging. ${ }^{31-33}$ In a recent prospective multi-institutional study, $74 \mathrm{hmCRC}$ patients who had previously been treated with at least two or three lines of systemic chemotherapy were randomized to receive DEBIRI-TACE or systemic FOLFIRI. ${ }^{33}$ Subjects in the DEBIRI-
TACE group showed gains in overall survival (7 months) and progression-free survival ( 3 months) compared with those who received systemic FOLFIRI. Achieving such results can be challenging, however, because the recommended treatment algorithm for TACE with DEBIRI requires two treatment sessions for each involved lobe separated by 4 weeks. $^{34,35}$ Thus hmCRC patients with bilobar disease, which are the majority in the salvage setting, need to undergo four procedures. Furthermore, the DEBIRI toxicity data suggest a more severe postembolization syndrome relative to RE, with grade 2 to 3 abdominal pain observed in up to $30 \%$ of treatment sessions. ${ }^{33,34}$

Conventional TACE is another option for the treatment of unresectable hmCRC. ${ }^{36-40}$ There have been no studies directly comparing DEBIRI-TACE and conventional TACE; thus the decision to provide one over the other is often a matter of institutional preference. We currently prefer DEBIRI-TACE because of the encouraging results of recent clinical trials, the accepted role of irinotecan in frontline systemic regimens for metastatic CRC, and the paucity of compelling data favoring conventional TACE despite several decades of clinical experience.

HAI requires implantation of a pump and an attached catheter placed through the gastroduodenal artery (GDA) and tip positioned at the GDA-hepatic artery junction for repeated intra-arterial delivery of various chemotherapy agents, most frequently floxuridine (FUdR). ${ }^{9,41}$ Placement of HAI systems is traditionally performed surgically with surgical skeletonization of the hepatic artery, although percutaneous techniques using arterial ports have also been reported. ${ }^{42,43}$ PHP is similar to HAI except that a concentrated chemotherapeutic (melphalan) is delivered via a percutaneously placed hepatic artery catheter, and a second catheter with occlusion balloons inflated in the supra- and infrahepatic inferior vena cava is used to isolate and collect hepatic venous drainage that is then filtered prior to return to the circulation. The PHP organ isolation delivery system (CHEMOSAT; Delcath Systems, New York, NY) is currently not approved by the Food and Drug Administration (FDA) for use in the United States. Availability of both HAI and PHP is limited to a few centers in the United States, and readers are referred to other sources for more details. ${ }^{9,41,44,45}$

\section{${ }^{90} \mathrm{Y}$ Radioembolization}

There are currently two commercially available ${ }^{90}$ Y-labeled microspheres in the United States, with one composed of a biocompatible resin (SIR-Spheres; SIRTex Medical, Ltd., Sydney, Australia) and the other composed of glass (TheraSphere; MDS Nordion, Inc., Ontario, Canada). The resin microspheres have full premarket approval by the FDA for the treatment of unresectable hmCRC. ${ }^{46}$ The glass microspheres have a Humanitarian Device Exception from the FDA for treatment of unresectable hepatocellular carcinoma. ${ }^{47}$ In addition to these regulatory approval (and thus institutional review board and insurance coverage) differences, we use the resin ${ }^{90} \mathrm{Y}$ microspheres for most hmCRC cases because only a small minority of patients in the hmCRC RE literature were treated with glass microspheres. ${ }^{14}$ We do, however, use the 

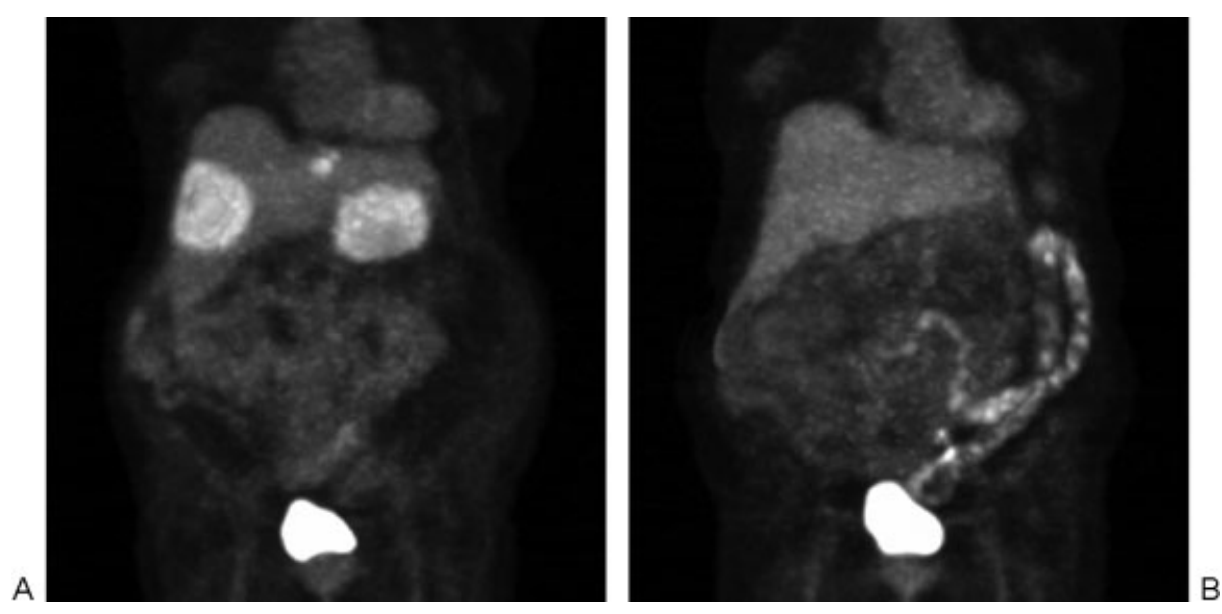

Figure 1 Complete response to first-line treatment with radioembolization (RE) using yttrium-90 $\left({ }^{90} \mathrm{Y}\right)$ resin microspheres. (A) Frontal maximum intensity projection of positron emission tomography scan of a patient with liver metastases from colorectal cancer prior to treatment demonstrated large bilobar hypermetabolic hepatic lesions. (B) Follow-up imaging studies 3 and 6 months after whole-liver RE treatment showed complete response of the metastases, which was sustained 1 year later while the patient was receiving systemic chemotherapy.

less embolic glass microspheres in cases of portal vein thrombosis, previous portal vein embolization, or severe hepatic arteriopathy due to bevacizumab or other systemic vasculotoxins. Portal vein compromise is a contraindication for the resin microspheres, which are embolic due to their larger diameter (20 to $60 \mu \mathrm{m}$ versus 20 to $30 \mu \mathrm{m}$ ) and lower per particle radioactivity ( $50 \mathrm{~Bq}$ versus $2500 \mathrm{~Bq}$ ) that requires delivery of a substantially greater number of microspheres to achieve any given activity. ${ }^{46,47}$

\section{Preprocedure}

The work-up for ${ }^{90} \mathrm{Y}$ RE includes review of recent crosssectional imaging, either multiphasic contrast-enhanced computed tomography (CT) or magnetic resonance imaging as well as positron emission tomography (PET) if available. This evaluation is performed to assess intrahepatic tumor burden and distribution, extrahepatic disease, portal vein patency, and hepatic arterial anatomy. Clinical laboratory tests are obtained to measure hepatic, renal, and hematopoietic function, and to determine a baseline carcinoembryonic antigen (CEA) level. All patients are seen in clinic where clinical history, CRC treatment history, physical examination, and performance status are evaluated. The clinic visit is also used to discuss with patients their candidacy for RE, the treatment process, expected outcomes, potential toxicities, radiation safety, and postprocedure follow-up. Patients are provided an informational package that contains a DVD on $\mathrm{RE}^{48}$ and prescriptions for medications and laboratory tests to be used following ${ }^{90} \mathrm{Y}$ microsphere administration.

In our practice, selection criteria for treatment of CRC patients with ${ }^{90} \mathrm{Y}$ RE include unresectable metastatic disease isolated to or mainly involving the liver, Eastern Cooperative Oncology Group (ECOG) Performance Status (PS) ${ }^{49} \leq 2$, bilirubin $<2.0 \mathrm{mg} / \mathrm{dL}$, and the absence of uncorrectable coagulopathy, renal function, or allergy issues that would preclude arterial catheterization or iodinated contrast angiography. We recommend RE only for patients in whom the hepatic disease is the most life threatening, and thus we do not perform RE on patients with brain metastases, extensive peritoneal metastases, debilitating lung or bone metastases, and so on. Furthermore, patients with a compromised ampulla of Vater, whether due to previous biliary surgery or biliary stent placement, are at high risk for infectious complications and should undergo antibiotic prophylaxis prior to and following this treatment. ${ }^{50}$ Patients with biliary obstruction may undergo endoscopic or percutaneous drainage and

Table 1 Outcomes of Radioembolization with ${ }^{90} \mathrm{Y}$ resin Microspheres for Salvage Therapy of Patients with Hepatic Metastases from Colorectal Cancer

\begin{tabular}{|c|c|c|c|c|}
\hline Study & $N$ & ORR (\%) & SD (\%) & Median overall survival (months) \\
\hline Kennedy et $\mathrm{al}^{22}$ & 208 & 35.5 & 54.8 & $\begin{array}{l}10.5 \text { (responders) } \\
4.5 \text { (nonresponders) }\end{array}$ \\
\hline Bester et $\mathrm{a}^{23}$ & 224 & NR & NR & 11.9 \\
\hline Cosimelli et al $^{21}$ & 50 & 24.0 & 24.0 & 12.6 \\
\hline Nace et $\mathrm{al}^{26}$ & 51 & 12.9 & 64.5 & 10.2 \\
\hline Cianni et a ${ }^{24}$ & 41 & 46.3 & 36.2 & 11.6 \\
\hline Jakobs et $\mathrm{a}^{25}$ & 41 & 17.1 & 61.0 & 10.5 \\
\hline
\end{tabular}

Abbreviations: NR, not reported; ORR, objective response rate (complete response plus partial response); SD, stable disease. 
be reevaluated for candidacy after normalization of biliary physiology.

Salvage hmCRC patients tend to present with bilobar disease without underlying cirrhosis, and serum bilirubin is usually normal. However, in patients without biliary obstruction, liver function may be compromised due to extensive intrahepatic tumor burden or iatrogenic hepatic insults. Salvage patients typically have undergone numerous rounds of systemic chemotherapy before presenting for locoregional intra-arterial therapy, so it is imperative to obtain a thorough treatment history. Prior or ongoing systemic chemotherapy regimens with or without targeted biological agents, external-beam radiation therapy, hepatic surgery or ablation, or other intra-arterial therapies may have an impact on RE treatment planning, safety, and efficacy. Due to concern for potential increased risk of vascular injury and attenuated arteries that may lead to early reflux and nontarget embolization, bevacizumab is held for 4 to 6 weeks before the RE process. Radiosensitizing chemotherapies are typically held for at least 2 weeks to avoid superimposition of toxicities, but this practice may change as new data on combination therapies become available.

\section{Preparatory Angiography and Technetium 99m- Macroaggregated Albumin Scintigraphy}

The RE process is divided into two outpatient sessions performed up to 2 weeks apart. The overall goals of the first preparatory session are twofold: to skeletonize the hepatic arterial vasculature to ensure targeted delivery of ${ }^{90} \mathrm{Y}$ microspheres to tumor-bearing liver but not to the gastrointestinal tract, where inadvertent nontarget deposition can result in ulceration, ${ }^{51}$ and then to quantify hepatopulmonary shunting by technetium $99 \mathrm{~m}\left({ }^{99 \mathrm{~m}} \mathrm{Tc}\right)$-labeled macroaggregated albumin (MAA) scintigraphy. Meticulous angiography is performed to characterize the hepatic arterial supply, assess flow dynamics, identify hepaticoenteric arterial communications, and recognize tumor-supplying collateral vessels para- sitized from extrahepatic arteries (EHAs). Endovascular isolation or skeletonization of the hepatic arterial vasculature is then performed by aggressive prophylactic embolization of visualized hepaticoenteric anastomoses to prevent nontarget gastrointestinal delivery. Parasitized EHAs and select variant hepatic arteries can be occluded to restore and consolidate intrahepatic hepatic artery inflow (-Fig. 2)..$^{52,53}$

The evaluation process begins with careful review of crosssectional imaging to identify hepatic artery anatomical variants and to screen for potential parasitized EHAs. After femoral access is achieved, an abdominal aortogram is performed with injection of contrast at around the T8 level to map the hepatic arterial anatomy and to reveal hypertrophied EHAs suspicious for parasitization. Commonly parasitized EHAs include the right inferior phrenic (-Fig. 2) and right T8 to T11 intercostal arteries. ${ }^{53}$ After a parasitized EHA is confirmed by selective arteriography, it may be embolized to stasis using large particles with or without blockade of the parent EHA with coils. The purpose of this is not to induce tumor ischemia, but to restore intrahepatic arterial supply to territories previously perfused by the parasitized EHA through preexisting intrahepatic channels to allow for hepatic artery delivery of ${ }^{90} \mathrm{Y}$ microspheres to these regions.

Digital subtraction angiography (DSA) is performed with a $5 \mathrm{~F}$ catheter positioned in the common hepatic artery to carefully identify vessels that may supply extrahepatic parenchyma ( - Fig. 3 ). We routinely coil embolize the right gastric artery (RGA) and the GDA, unless planned selective administration of ${ }^{90} \mathrm{Y}$ microspheres is far distal to these vessels. Hepatic arteriography is often performed in at least two projections to better visualize the origin of the RGA, which most commonly arises from the proximal left or proper hepatic artery. Cannulation of the RGA can be challenging, requiring use of a preshaped microcatheter, formation of a Waltman loop, or retrograde catheterization via the left gastric artery. ${ }^{54}$ For both the RGA and GDA, we aim to embolize the vessel completely to its origin because small
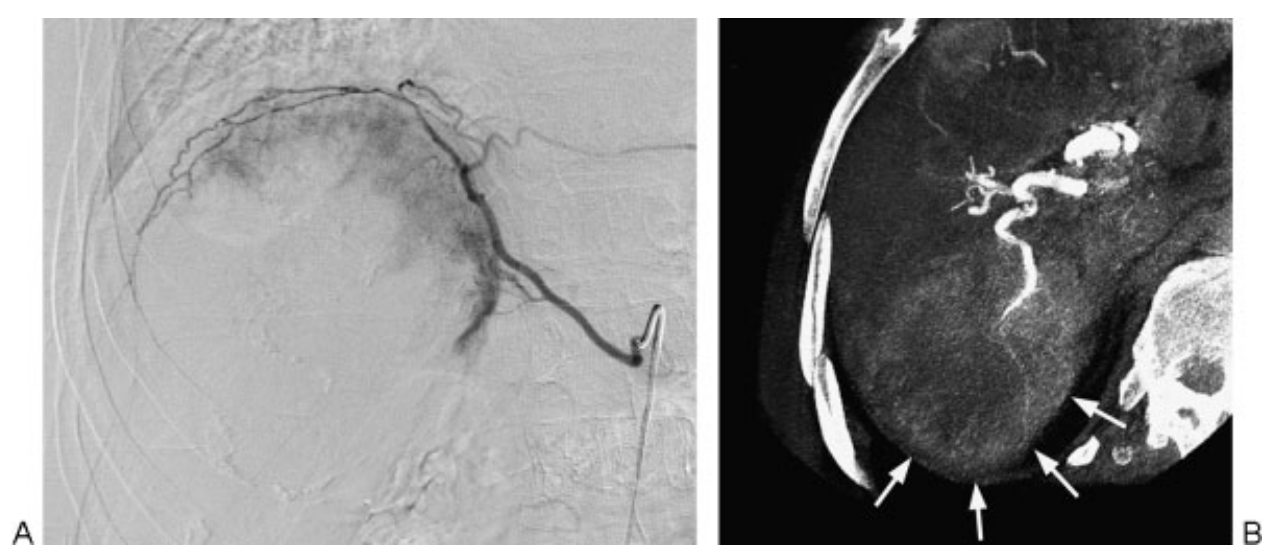

Figure 2 Embolization of parasitized extrahepatic arteries (EHAs). (A) Arteriogram of the right inferior phrenic artery demonstrated parasitized collateral vessels supplying the posterior superior medial periphery of a large hepatic colorectal cancer metastasis via the bare area. After prophylactic coil embolization of a left phrenic arterial branch, the tumor-supplying parasitized branches were embolized to stasis using 500 - to 700- $\mu \mathrm{m}$ Embospheres (Merit Medical Systems, Inc., South Jordan, UT). (B) Subsequent cone-beam C-arm computed tomography performed with contrast administered via the common hepatic artery demonstrated contrast enhancement of the entire posterior right hepatic lobe lesion all the way to the capsule, confirming restoration of intrahepatic arterial supply to portions of the lesion previously supplied by parasitized EHAs (arrows). 

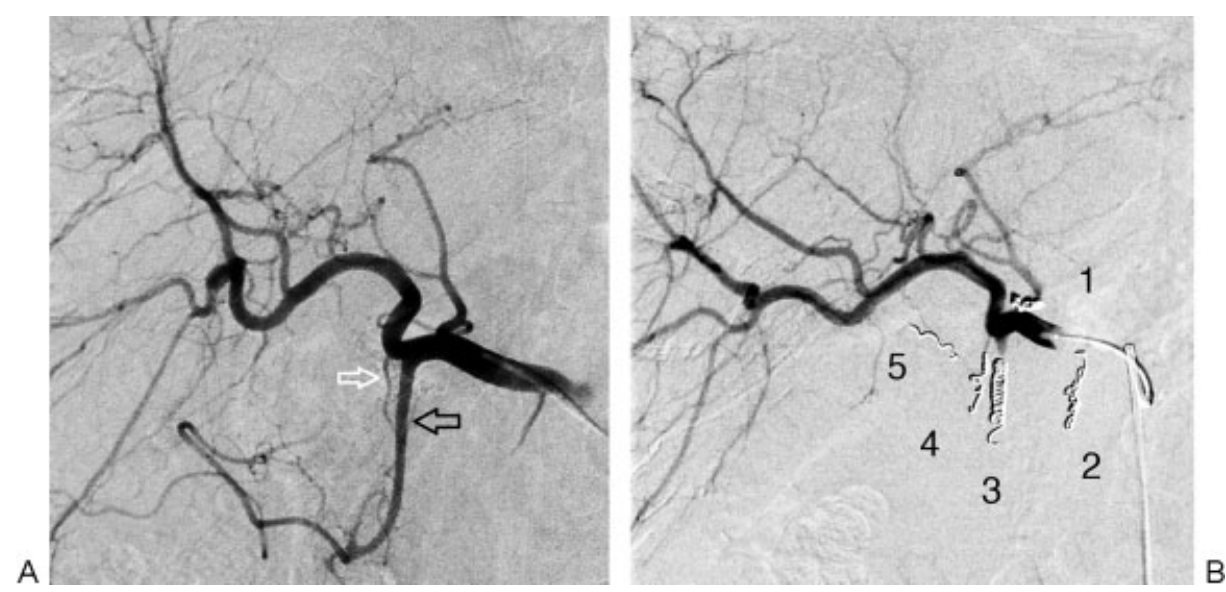

Figure 3 Hepatic artery skeletonization in preparation for radioembolization (RE). (A) Common hepatic arteriogram portrayed numerous hepaticoenteric communications, as well as anatomical variants of the gastroduodenal (black arrow) and posterior superior pancreaticoduodenal (white arrow) arteries originating from the right hepatic artery. (B) Endovascular skeletonization was performed by prophylactic coil embolization of all recognized hepaticoenteric anastomoses including the right gastric artery (1), dorsal pancreatic artery (2), gastroduodenal artery (3), posterior superior pancreaticoduodenal artery (4), and a supraduodenal artery originating from the right hepatic artery (5). RE was performed by separate microsphere administrations via the left and right main hepatic arteries.

proximal branches may hypertrophy and continue to supply the gastrointestinal tract if embolization is incomplete. Accurate embolization of the origin can be facilitated by use of detachable microcoils or AMPLATZER Vascular Plugs (St. Jude Medical, Inc., St. Paul, MN). Other hepaticoenteric anastomoses that we commonly embolize include the supraduodenal, pancreaticoduodenal (retroduodenal), and falciform arteries. Angiographic images of the left hepatic artery should be carefully reviewed for the presence of accessory left gastric, accessory phrenic, and inferior esophageal arteries. These should be coil embolized if identified. Prophylactic embolization of the cystic artery is controversial. Doing so does not appear to decrease the incidence of surgical cholecystitis ( $\sim 1 \%$ ); thus we only embolize the cystic artery if a bowelsupplying marginal or supraduodenal branch is identified originating from the cystic artery.

For patients with replaced or accessory hepatic arteries, each variant hepatic artery is evaluated angiographically to characterize the hepatic territory supplied. In situations where the planned treatment territory is supplied by both a variant and nonvariant hepatic artery, the less dominant supplying vessel may be coil embolized to consolidate hepatic arterial inflow and thus simplify ${ }^{90} \mathrm{Y}$ microsphere administration. $^{52}$ Inflow consolidation can also improve safety by avoiding microsphere infusion into variant hepatic arteries that originate in close proximity to enteric branches, where reflux would result in gastrointestinal complications.

To determine the completeness of hepatic artery skeletonization and to evaluate where ${ }^{90} \mathrm{Y}$ microspheres will distribute in the liver, a microcatheter is positioned in the anticipated microsphere implantation site(s) to perform contrast-enhanced cone-beam C-arm CT (CACT) using an imaging protocol previously described. ${ }^{55}$ If treatment of the whole liver is planned in a patient with standard hepatic arterial anatomy, contrast is administered via the proper hepatic artery. For planned lobar RE, contrast is injected through the respective lobar hepatic artery. CACT images are carefully scrutinized for possible extrahepatic parenchymal enhancement and areas of nonenhancement in the desired intrahepatic treatment territory. The former indicates the presence of unrecognized hepaticoenteric communications, whereas the latter signifies competitive intrahepatic perfusion by undetected accessory hepatic arteries or parasitized EHAs. We have found that CACT provides valuable arterial perfusion information not detectable by DSA or ${ }^{99 \mathrm{~m}} \mathrm{Tc}-\mathrm{MAA}$ scintigraphy, and we frequently use this technology throughout the preparatory angiography session as a problemsolving tool to interrogate vessels in which it is unclear what tissue is being perfused. ${ }^{55}$

Once prophylactic embolization is satisfactorily completed, $1 \mathrm{mCi}$ of ${ }^{99 \mathrm{~m}} \mathrm{Tc}-\mathrm{MAA}$ is administered intra-arterially through a catheter positioned at the planned microsphere implantation site(s), as previously described for completion contrast-enhanced CACT. Patients then undergo planar scintigraphy to calculate lung shunt fraction (LSF) and singlephoton emission CT (SPECT) imaging to provide a second lower resolution means to evaluate for possible extrahepatic deposition and predict intrahepatic microsphere distribution. Unique to our practice, sulfur colloid (SC) imaging is also performed. Without moving the patient, $5 \mathrm{mCi}$ of ${ }^{99 \mathrm{~m}} \mathrm{Tc}$-SC is administered intravenously, and SPECT is repeated. ${ }^{56}$ The ${ }^{99 \mathrm{~m}} \mathrm{Tc}-\mathrm{MAA}$ and ${ }^{99 \mathrm{~m}} \mathrm{Tc}-\mathrm{SC}$ SPECT data sets are fused and analyzed to better characterize predicted radiation dose distribution between normal liver and tumor.

\section{Dosimetry}

For resin ${ }^{90} \mathrm{Y}$ microspheres, the standard body surface area (BSA) method is used to calculate dose. Activity is determined using the following formulas: Activity in $\mathrm{GBq}=(\mathrm{BSA}-0.2)+$ (\% tumor involvement); BSA in $\mathrm{m}^{2}=0.20247 \times$ (height in $\mathrm{m})^{0.725} \times(\text { weight in } \mathrm{kg})^{0.425}$. Percent tumor involvement is estimated according to preprocedure cross-sectional 
imaging and preparatory angiography and CACT images. Although the BSA method is recommended by consensus and by the manufacturing company, ${ }^{57}$ it has recognized limitations. ${ }^{58}$ Improving and customizing dose calculation is the subject of ongoing investigations.

Per manufacturer instructions, to minimize the risk of radiation pneumonitis, dose reduction should be considered when LSF is between $10 \%$ and $20 \%$; RE should not be performed when LSF is $>20 \% .{ }^{46}$ However, we prefer to calculate the expected pulmonary absorbed dose and make adjustments only if the exposure to the lungs is $>30 \mathrm{~Gy}$ per treatment or $50 \mathrm{~Gy}$ cumulatively. ${ }^{59}$ Fortunately, elevated LSF is rare in hmCRC patients.

We also incorporate further dose adjustments or potentially fractionate treatment in hmCRC patients who are candidates for RE but are at higher risk for adverse events. Such situations may include an ECOG PS of 2, total bilirubin level of 1.5 to $2 \mathrm{mg} / \mathrm{dL}$, advanced age, multiple lines of prior hepatotoxic systemic chemotherapy, previous resections or ablations, and history of external-beam radiation therapy that overlapped the hepatic region. ${ }^{60}$

\section{${ }^{90} \mathrm{Y}$ Microsphere Administration}

To begin the ${ }^{90} \mathrm{Y}$ microsphere administration procedure, DSA and contrast-enhanced CACT are repeated to confirm completeness of hepatic artery skeletonization. In our experience, $\sim 30 \%$ of treatment sessions reveal new or recanalized hepaticoenteric vessels. ${ }^{61}$ If these communications persist and are too diminutive or tortuous to coil embolize, a special antireflux microcatheter (Surefire Infusion System; Surefire Medical, Inc., Westminster, CO) may be positioned distal to these vessels for ${ }^{90} \mathrm{Y}$ microsphere administration. ${ }^{62}$ Otherwise, standard microcatheters are used for microsphere delivery.

For patients with bilobar disease, whole liver treatment may be performed in a single session or in two sequential lobar treatments at different sessions separated by 4 to 6 weeks. Whole liver single session treatment is preferred in part to prevent interval accelerated growth of contralateral untreated tumors and also to avoid potential medical insurance difficulties. To mitigate the risk for gastrointestinal complications, when feasible, single-session whole liver treatments are performed with sequential administrations via lobar arteries rather than delivery via the common or proper hepatic artery. For each infusion site, a separate microsphere dose is drawn and delivered.

Before microsphere delivery, a corticosteroid (dexamethasone 8 to $12 \mathrm{mg}$ ), a proton pump inhibitor (pantoprazole $40 \mathrm{mg}$ ), and an antiemetic (ondansetron $8 \mathrm{mg}$ ) are administered intravenously. Readers are referred to the package insert for detailed instructions on how to set up and use the device-specific administration apparatus. ${ }^{46,47}$ After confirming correct positioning of the microcatheter and presence of antegrade arterial flow, the ${ }^{90} \mathrm{Y}$ microspheres are slowly delivered in small aliquots. Because the resin ${ }^{90} \mathrm{Y}$ microspheres are embolic, there is risk of reflux, and thus it is important to monitor delivery carefully with iterative angiography between aliquots. If near-stasis of antegrade flow is observed, microsphere infusion should be suspended and possibly terminated. After ${ }^{90} \mathrm{Y}$ microsphere implantation, some groups advocate performing a Bremsstrahlung or PET scan to document a low-resolution image of microsphere distribution. ${ }^{63}$ The value of this practice has yet to be proven because there are no methods to reverse nontarget microsphere administration.

\section{Postprocedure and Follow-Up}

After microspheres are delivered, patients are discharged the same day with a 10-day oral methylprednisolone taper regimen, 30-day course of proton pump inhibitor, and oral analgesic and antiemetic agents as needed. Toxicity is usually mild, featuring fatigue, abdominal discomfort, nausea, and anorexia with symptoms typically resolving in 1 to 4 weeks. A follow-up telephone call to patients is made 5 to 7 days after treatment to identify potential severe toxicities and to provide needed encouragement.

Clinical laboratory tests (complete blood count with differential, liver function tests, coagulation panel, and CEA level) are obtained at 2, 4, 8, and 12 weeks postprocedure, and follow-up cross-sectional imaging is performed in 2 to 3 months. Patients are seen in clinic 1 month after ${ }^{90} \mathrm{Y}$ microsphere administration to assess for potential complications, and then after follow-up cross-sectional imaging is obtained to review outcomes.

\section{TACE with DEBIRI}

Although DEBIRI-TACE is being proposed as a possible competitor to RE, the state of the published literature is limited. This intra-arterial hmCRC treatment should certainly be considered in cases where ${ }^{90} \mathrm{Y}$ RE is contraindicated or if there is recurrent or residual disease following RE. Patients with $>60 \%$ metastatic replacement of the liver are cautioned against this treatment. ${ }^{35}$ Selection criteria are otherwise similar to those used for RE and conventional TACE.

For hmCRC patients with bilobar disease, the recommended treatment algorithm requires four separate lobar treatments, alternating between right and left lobes with at least a 2-week interval between sessions. ${ }^{34,35}$ For each treatment session, up to one vial of 100 - to $300-\mu$ m LC Beads (Biocompatibles, Oxford, CT) loaded with 100-mg irinotecan is delivered, as selectively as appropriate to treat all the visible hepatic disease.

DEBIRI-TACE is performed in a similar fashion to other TACE procedures, ${ }^{64,65}$ with the main difference being the use of more aggressive analgesic measures because many patients experience more severe abdominal pain compared with patients who undergo conventional TACE. ${ }^{33,34}$ In the preoperative area, patients are provided the same prophylactic drugs as with RE, including antiemetics, dexamethasone, and a proton pump inhibitor. In addition, a patient-controlled analgesia (PCA) pump is prepared for postprocedure use. After the hepatic arterial anatomy is evaluated angiographically, a microcatheter is positioned at the desired location for selective bead delivery. The supernatant containing free irinotecan is removed, and the loaded beads are suspended 
in $\sim 20 \mathrm{~mL}$ of nonionic contrast. Before administration of the beads, 2 to $4 \mathrm{~mL}$ of $1 \%$ preservative-free lidocaine may be slowly injected intra-arterially to diminish potential TACErelated pain. ${ }^{66}$ The beads are then slowly delivered under fluoroscopic imaging to monitor for reflux. Postprocedure, patients are admitted overnight with the PCA pump and antiemetic medications as needed for management of postembolization syndrome, and they are typically discharged the next day. After discharge, patients are prescribed a 30-day course of a proton pump inhibitor and oral analgesic and antiemetic agents as needed.

\section{Conclusions}

Based on the current literature on intra-arterial therapies for unresectable hmCRC, appropriate candidates are treated with ${ }^{90} \mathrm{Y}$ RE using resin microspheres, usually in the salvage setting. Given the less mature data for DEBIRI-TACE, this treatment modality is currently reserved for patients who have contraindications to $\mathrm{RE}$ or present with residual or recurrent metastatic disease after $\mathrm{RE}$. The role of intraarterial therapies will continue to evolve and further integrate into the treatment algorithm for hmCRC as level I data become available. There is considerable optimism for the use of ${ }^{90} \mathrm{Y}$ RE earlier in the disease process and with concomitant radiosensitizing chemotherapy regimens as frontline treatment for hmCRC. ${ }^{15-20}$ Several prospective clinical trials combining RE with chemotherapy regimens and/or biological agents are currently underway, and they will undoubtedly affect the way interventional radiologists care for patients with metastatic CRC. ${ }^{9,12}$

\section{References}

1 Jemal A, Bray F, Center MM, Ferlay J, Ward E, Forman D. Global cancer statistics. CA Cancer J Clin 2011;61(2):69-90

2 Surveillance Research Program, National Cancer Institute. SEER stat fact sheets: Colon and rectum. Available at: http://seer.cancer. gov/statfacts/html/colorect.html. Accessed August 20, 2012

3 Weiss L, Grundmann E, Torhorst J, et al. Haematogenous metastatic patterns in colonic carcinoma: an analysis of 1541 necropsies. J Pathol 1986;150(3):195-203

4 Choti MA. Surgical resection of hepatic metastases. In: Geschwind JF, Soulen MC, eds. Interventional Oncology: Principles and Practice. New York, NY: Cambridge University Press; 2008:243248

5 Sasson AR, Sigurdson ER. Surgical treatment of liver metastases. Semin Oncol 2002;29(2):107-118

6 Ma WW, Messersmith WA. Medical management of colorectal liver metastasis. In: Geschwind JF, Soulen MC, eds. Interventional Oncology: Principles and Practice. New York, NY: Cambridge University Press; 2008:222-242

7 Köhne CH, Lenz HJ. Chemotherapy with targeted agents for the treatment of metastatic colorectal cancer. Oncologist 2009;14(5): 478-488

8 Breedis C, Young G. The blood supply of neoplasms in the liver. Am J Pathol 1954;30(5):969-977

9 de Baere T, Deschamps F. Arterial therapies of colorectal cancer metastases to the liver. Abdom Imaging 2011;36(6):661-670

10 Wasan H, Kennedy A, Coldwell D, Sangro B, Salem R. Integrating radioembolization with chemotherapy in the treatment paradigm for unresectable colorectal liver metastases. Am J Clin Oncol 2012; 35(3):293-301

11 Salem R, Thurston KG. Radioembolization with yttrium-90 microspheres: a state-of-the-art brachytherapy treatment for primary and secondary liver malignancies: part 3: comprehensive literature review and future direction. J Vasc Interv Radiol 2006;17 (10):1571-1593

12 Nicolay NH, Berry DP, Sharma RA. Liver metastases from colorectal cancer: radioembolization with systemic therapy. Nat Rev Clin Oncol 2009;6(12):687-697

13 Deleporte A, Flamen P, Hendlisz A. State of the art: radiolabeled microspheres treatment for liver malignancies. Expert Opin Pharmacother 2010;11(4):579-586

14 Vente MA, Wondergem M, van der Tweel I, et al. Yttrium-90 microsphere radioembolization for the treatment of liver malignancies: a structured meta-analysis. Eur Radiol 2009;19(4): 951-959

15 Gulec SA, Pennington K, Wheeler J, et al. Yttrium-90 microsphereselective internal radiation therapy with chemotherapy (ChemoSIRT) for colorectal cancer liver metastases: an in vivo double-armcontrolled phase II trial. Am J Clin Oncol 2012; June 14 ( Epub ahead of print)

16 Sharma RA, Van Hazel GA, Morgan B, et al. Radioembolization of liver metastases from colorectal cancer using yttrium-90 microspheres with concomitant systemic oxaliplatin, fluorouracil, and leucovorin chemotherapy. J Clin Oncol 2007;25(9): 1099-1106

17 Van Hazel G, Blackwell A, Anderson J, et al. Randomised phase 2 trial of SIR-Spheres plus fluorouracil/leucovorin chemotherapy versus fluorouracil/leucovorin chemotherapy alone in advanced colorectal cancer. J Surg Oncol 2004;88(2):78-85

18 Kosmider S, Tan TH, Yip D, Dowling R, Lichtenstein M, Gibbs P. Radioembolization in combination with systemic chemotherapy as first-line therapy for liver metastases from colorectal cancer. J Vasc Interv Radiol 2011;22(6):780-786

19 van Hazel GA, Pavlakis N, Goldstein D, et al. Treatment of fluorouracil-refractory patients with liver metastases from colorectal cancer by using yttrium-90 resin microspheres plus concomitant systemic irinotecan chemotherapy. J Clin Oncol 2009;27(25): 4089-4095

20 Lim L, Gibbs P, Yip D, et al. A prospective evaluation of treatment with Selective Internal Radiation Therapy (SIR-spheres) in patients with unresectable liver metastases from colorectal cancer previously treated with 5-FU based chemotherapy. BMC Cancer 2005; $5: 132$

21 Cosimelli M, Golfieri R, Cagol PP, et al; Italian Society of Locoregional Therapies in Oncology (SITILO). Multi-centre phase II clinical trial of yttrium-90 resin microspheres alone in unresectable, chemotherapy refractory colorectal liver metastases. Br J Cancer 2010;103(3):324-331

22 Kennedy AS, Coldwell D, Nutting C, et al. Resin 90Y-microsphere brachytherapy for unresectable colorectal liver metastases: modern USA experience. Int J Radiat Oncol Biol Phys 2006;65 (2):412-425

23 Bester L, Meteling B, Pocock N, et al. Radioembolization versus standard care of hepatic metastases: comparative retrospective cohort study of survival outcomes and adverse events in salvage patients. J Vasc Interv Radiol 2012;23(1):96-105

24 Cianni R, Urigo C, Notarianni E, et al. Selective internal radiation therapy with SIR-spheres for the treatment of unresectable colorectal hepatic metastases. Cardiovasc Intervent Radiol 2009;32(6): 1179-1186

25 Jakobs TF, Hoffmann RT, Dehm K, et al. Hepatic yttrium-90 radioembolization of chemotherapy-refractory colorectal cancer liver metastases. J Vasc Interv Radiol 2008;19(8):1187-1195

26 Nace GW, Steel JL, Amesur N, et al. Yttrium-90 radioembolization for colorectal cancer liver metastases: a single institution experience. Int J Surg Oncol 2011;2011:571261 
27 Seidensticker R, Denecke T, Kraus P, et al. Matched-pair comparison of radioembolization plus best supportive care versus best supportive care alone for chemotherapy refractory liver-dominant colorectal metastases. Cardiovasc Intervent Radiol 2012;35 (5):1066-1073

28 Hendlisz A, Van den Eynde M, Peeters M, et al. Phase III trial comparing protracted intravenous fluorouracil infusion alone or with yttrium-90 resin microspheres radioembolization for liverlimited metastatic colorectal cancer refractory to standard chemotherapy. J Clin Oncol 2010;28(23):3687-3694

29 Lewandowski RJ, Thurston KG, Goin JE, et al. 90Y microsphere (TheraSphere) treatment for unresectable colorectal cancer metastases of the liver: response to treatment at targeted doses of $135-150 \mathrm{~Gy}$ as measured by [18F]fluorodeoxyglucose positron emission tomography and computed tomographic imaging. J Vasc Interv Radiol 2005;16(12):1641-1651

30 Aliberti C, Tilli M, Benea G, Fiorentini G. Trans-arterial chemoembolization (TACE) of liver metastases from colorectal cancer using irinotecan-eluting beads: preliminary results. Anticancer Res 2006;26(5B):3793-3795

31 Aliberti C, Fiorentini G, Muzzio PC, et al. Trans-arterial chemoembolization of metastatic colorectal carcinoma to the liver adopting DC Bead ${ }^{\circledR}$, drug-eluting bead loaded with irinotecan: results of a phase II clinical study. Anticancer Res 2011;31 (12):4581-4587

32 Martin RC, Joshi J, Robbins K, et al. Hepatic intra-arterial injection of drug-eluting bead, irinotecan (DEBIRI) in unresectable colorectal liver metastases refractory to systemic chemotherapy: results of multi-institutional study. Ann Surg Oncol 2011;18(1): 192-198

33 Fiorentini G, Aliberti C, Tilli M, et al. Intra-arterial infusion of irinotecan-loaded drug-eluting beads (DEBIRI) versus intravenous therapy (FOLFIRI) for hepatic metastases from colorectal cancer: final results of a phase III study. Anticancer Res 2012;32(4): 1387-1395

34 Martin RC, Howard J, Tomalty D, et al. Toxicity of irinotecan-eluting beads in the treatment of hepatic malignancies: results of a multiinstitutional registry. Cardiovasc Intervent Radiol 2010;33(5): 960-966

35 Biocompatibles. DEBIRI algorithm-colorectal metastases. Available at: http://www.biocompatibles.com/products/dcbead/technique-guidelines. Accessed August 15, 2012

36 Albert M, Kiefer MV, Sun W, et al. Chemoembolization of colorectal liver metastases with cisplatin, doxorubicin, mitomycin C, Ethiodol, and polyvinyl alcohol. Cancer 2011;117(2):343-352

37 Vogl TJ, Gruber T, Balzer JO, Eichler K, Hammerstingl R, Zangos S. Repeated transarterial chemoembolization in the treatment of liver metastases of colorectal cancer: prospective study. Radiology 2009;250(1):281-289

38 Tellez C, Benson AB III, Lyster MT, et al. Phase II trial of chemoembolization for the treatment of metastatic colorectal carcinoma to the liver and review of the literature. Cancer 1998;82(7): 1250-1259

39 Sanz-Altamira PM, Spence LD, Huberman MS, et al. Selective chemoembolization in the management of hepatic metastases in refractory colorectal carcinoma: a phase II trial. Dis Colon Rectum 1997;40(7):770-775

40 Lang EK, Brown CL Jr. Colorectal metastases to the liver: selective chemoembolization. Radiology 1993;189(2):417-422

41 Kingham TP, D'Angelica M, Kemeny NE. Role of intra-arterial hepatic chemotherapy in the treatment of colorectal cancer metastases. J Surg Oncol 2010;102(8):988-995

42 Arai Y, Takeuchi Y, Inaba Y, et al. Percutaneous catheter placement for hepatic arterial infusion chemotherapy. Tech Vasc Interv Radiol 2007;10(1):30-37

43 Ganeshan A, Upponi S, Hon LQ, Warakaulle D, Uberoi R. Hepatic arterial infusion of chemotherapy: the role of diagnostic and interventional radiology. Ann Oncol 2008;19(5):847-851
44 Antoine RA. Technical considerations in percutaneous hepatic perfusion-a multi-center experience. J Extra Corpor Technol 2011;43(1):30-33

45 Miao N, Pingpank JF, Alexander HR, Steinberg SM, Beresneva T, Quezado ZM. Percutaneous hepatic perfusion in patients with metastatic liver cancer: anesthetic, hemodynamic, and metabolic considerations. Ann Surg Oncol 2008;15(3):815-823

46 SIRTeX Medical. SIR-Spheres Yttrium-90 microspheres package insert, October 2011. Available at: http://www.sirtex.com/files/ SSL-US-09.pdf. Accessed August 15, 2012

47 Nordion MDS. TheraSphere Yttrium-90 microspheres package insert, US ed. Available at: http://www.nordion.com/therasphere/ physicians-package-insert/package-insert-us.pdf. Accessed August 15,2012

48 Stanford Interventional Radiology. Interventional Oncology-Radioembolization. Available at: http://www.youtube.com/watch? v=3WwSfGPQq9g. Accessed August 15, 2012

49 Oken MM, Creech RH, Tormey DC, et al. Toxicity and response criteria of the Eastern Cooperative Oncology Group. Am J Clin Oncol 1982;5(6):649-655

50 Salem R, Thurston KG. Radioembolization with 90yttrium microspheres: a state-of-the-art brachytherapy treatment for primary and secondary liver malignancies. Part 2: special topics. J Vasc Interv Radiol 2006;17(9):1425-1439

51 Murthy R, Brown DB, Salem R, et al. Gastrointestinal complications associated with hepatic arterial yttrium-90 microsphere therapy. J Vasc Interv Radiol 2007;18(4):553-561; quiz 562

52 Abdelmaksoud MH, Louie JD, Kothary N, et al. Consolidation of hepatic arterial inflow by embolization of variant hepatic arteries in preparation for yttrium-90 radioembolization. J Vasc Interv Radiol 2011;22(10):1364-1371, e1

53 Abdelmaksoud MH, Louie JD, Kothary N, et al. Embolization of parasitized extrahepatic arteries to reestablish intrahepatic arterial supply to tumors before yttrium-90 radioembolization. J Vasc Interv Radiol 2011;22(10):1355-1362

54 Hashimoto M, Heianna J, Tate E, Kurosawa R, Nishii T, Mayama I. The feasibility of retrograde catheterization of the right gastric artery via the left gastric artery. J Vasc Interv Radiol 2001;12(9): 1103-1106

55 Louie JD, Kothary N, Kuo WT, et al. Incorporating cone-beam CT into the treatment planning for yttrium-90 radioembolization. J Vasc Interv Radiol 2009;20(5):606-613

56 Sze DY, Louie JD, Iagaru AH, Abdelmaksoud M, Goris ML. Survival after radioembolization is predicted by dose distribution scintigraphy fusion imaging [abstract]. J Vasc Interv Radiol 2010;21(2): S10

57 Kennedy A, Nag S, Salem R, et al. Recommendations for radioembolization of hepatic malignancies using yttrium-90 microsphere brachytherapy: a consensus panel report from the radioembolization brachytherapy oncology consortium. Int J Radiat Oncol Biol Phys 2007;68(1):13-23

58 Lam MG, Nijsen F, Smits M, et al. Limitations of the BSA-based dose calculation for Yttrium-90 radioembolization [abstract]. J Vasc Interv Radiol 2012;23(3):S68

59 Leung TW, Lau WY, Ho SK, et al. Radiation pneumonitis after selective internal radiation treatment with intraarterial 90yttrium-microspheres for inoperable hepatic tumors. Int J Radiat Oncol Biol Phys 1995;33(4):919-924

60 Abdelmaksoud M, Chang D, Eclov NC, et al. Safety of Y90 radioembolization in patients who have undergone previous external beam radiation therapy [abstract]. J Vasc Interv Radiol 2011; 22(3):S87

61 Abdelmaksoud MH, Hwang GL, Louie JD, et al. Development of new hepaticoenteric collateral pathways after hepatic arterial skeletonization in preparation for yttrium-90 radioembolization. J Vasc Interv Radiol 2010;21(9):1385-1395

62 Louie JD, Wang EA, Broadwell S, et al. First in man experience with the Surefire infusion system: a dedicated microcatheter system to 
Wang et al.

eliminate reflux during embolotherapy [abstract]. J Vasc Interv Radiol 2012;23(3):S80

63 Ahmadzadehfar H, Muckle M, Sabet A, et al. The significance of bremsstrahlung SPECT/CT after yttrium-90 radioembolization treatment in the prediction of extrahepatic side effects. Eur J Nucl Med Mol Imaging 2011;39(2):309-315

64 Ha BY, Ahmed A, Sze DY, et al. Long-term survival of patients with unresectable hepatocellular carcinoma treated with transcatheter arterial chemoinfusion. Aliment Pharmacol Ther 2007;26(6): 839-846
65 Tognolini A, Louie JD, Hwang GL, Hofmann LV, Sze DY, Kothary N. Utility of C-arm CT in patients with hepatocellular carcinoma undergoing transhepatic arterial chemoembolization. J Vasc Interv Radiol 2010;21(3):339-347

66 Fiorentini G, Aliberti C, Benea G, et al. TACE of liver metastases from colorectal cancer adopting irinotecan-eluting beads: beneficial effect of palliative intra-arterial lidocaine and post-procedure supportive therapy on the control of side effects. Hepatogastroenterology 2008;55(88):2077-2082 